\title{
\begin{tabular}{c|} 
Clinical Schizophrenia \\
\& Related Psychoses
\end{tabular}
}

\section{Emotional, cognitive and behavioral reactions to paranoid symptoms in clinical and nonclinical population}

\begin{tabular}{|r|l|}
\hline Journal: & Clinical Schizophrenia \& Related Psychoses \\
\hline Manuscript ID: & CSRP-02-14-0307.R1 \\
\hline Danuscript Type: & Original Contribution \\
\hline Complete List of Authors: & $\begin{array}{l}\text { Carvalho, Célia; University of Azores, Department of Educational Sciences; } \\
\text { University of Coimbra, CINEICC, Faculty of Psychology and Educational } \\
\text { Sciences } \\
\text { Pinto-Gouveia, José; University of Coimbra, CINEICC, Faculty of Psychology } \\
\text { and Educational Sciences } \\
\text { Bernardo Peixoto, Ermelindo; University of Azores, Department of } \\
\text { Educational Sciences } \\
\text { da Motta, Carolina; University of Azores, Department of Educational } \\
\text { Sciences; University of Coimbra, CINEICC, Faculty of Psychology and } \\
\text { Educational Sciences }\end{array}$ \\
\hline Keyword: & \begin{tabular}{l} 
schizophrenia, paranoia, cognitive behavioral therapy, delusions \\
\hline
\end{tabular} \\
\hline
\end{tabular}


RUNNING HEAD: Differences in reactions to paranoia

\title{
Emotional, cognitive and behavioral reactions to paranoid symptoms in clinical and nonclinical populations
}

\begin{abstract}
Background: Paranoia is a disruptive belief that can vary across a continuum, ranging from persecutory delusions presented in clinical settings to paranoid cognitions that are highly prevalent in the general population. The literature suggests that paranoid thoughts derive from the activation of a paranoid schema or information processing biases that can be sensitive to socially ambiguous stimuli and influence the processing of threatening situations. Method: Four groups (Schizophrenic participants in active psychotic phases, $n=6$; stable participants in remission, $n=30$; participants' relatives, $n=32$; and healthy controls, $n=64$ ) were assessed with self-report questionnaires to determine how the reactions to paranoia of clinical patients differ from healthy individuals. Cognitive, emotional and behavioral dimensions of their reactions to these paranoid thoughts were examined. Results: Paranoid individuals were present in all groups. Most participants referred the rejection by others as an important trigger of paranoid ideations, while active psychotic were unable to identify triggering situations to their thoughts and reactions. This may be determinant to the different reactions and the different degree of invalidation caused by paranoid thoughts observed across groups. Conclusion: Clinical and non-clinical expressions of paranoid ideations differ in terms of their cognitive, emotional and behavioral components. It is suggested that, in socially ambiguous situations, paranoid participants (presenting lower thresholds of paranoid schema activation) lose the opportunity to disconfirm their paranoid beliefs by resourcing to more maladaptive coping strategies. Consequently, by dwelling on these thoughts, the amount of time spent thinking about their condition and the disability related to the disease increases.
\end{abstract}

Keywords: paranoia, schizophrenia, cognitive-behavioral therapy, invalidation. 
RUNNING HEAD: Differences in reactions to paranoia

\section{Introduction}

Paranoid ideation has been regarded a continuous cognitive construct (1-6), as several studies have been supporting over the past decades (7-11). However, the link between clinical and sub-clinical paranoia and the etiology of paranoid beliefs is not completely understood. Paranoia has a significant impact on cognition and social behavior $(2,8,12,13)$. Studies have also shown the significant effects of persecutory delusions in attribution styles and cognitive reasoning tasks $(12,14,15)$. Further, current literature and research suggest that paranoid thoughts arise from the triggering of a paranoid schema or a bias in information processing that can be sensitive to socially ambiguous stimuli, and can influence the processing of threatening situations $(2,13,16-20)$. This bias is considered an important factor in the etiology and maintenance of persecutory delusions $(13,21,22)$.

Paranoia involves cognitive processes similar to those that occur in the normal experiences of self-consciousness, and can vary in a continuum, ranging from clinical symptoms, like persecutory delusions, to paranoid thoughts and behaviors that occur in individuals without psychopathology (23). Sub-clinical paranoia is characterized by an exaggerated self-referential bias that can occur in everyday life. Typically, there is a relatively stable tendency toward suspicion, feelings of resentment, lack of confidence and beliefs in external control and influence $(8,23)$. Such thoughts contrast with those prevailing in clinical paranoia, which include persecutory delusions and extreme lack of confidence. However, only a few studies have examined the social information processing aspects of paranoia (8).

Studies on personal paranoid experiences along different cognitive, behavioral and emotional dimensions paranoia in non-clinical samples (college students) have found that $47 \%$ to $70 \%$ of participants experienced paranoia (28). Additionally, high levels of paranoia 
RUNNING HEAD: Differences in reactions to paranoia

were more directly linked to emotional and avoidance coping strategies, than to rational strategies in non-clinical samples (11).

Previous studies concerning these issues $(11,13,23-29)$ were more narrowly focused on specific populations and less multidimensional in the assessment of paranoid ideation. We chose to evaluate paranoid schizophrenia in continuity with normal paranoia experiences as a way to study paranoid ideation and of clarifying its etiology. As such, it is important to explore the characteristics of paranoia in both healthy populations and in patients with schizophrenia, assessing different dimensions of their paranoid beliefs, and the degree of invalidation they caused. In a previous study (30), it was established that paranoid thoughts occur in continuity, and the degree of conviction and distress caused by paranoid beliefs vary among different groups of subjects. Results pointed out to active schizophrenic participants to be the most affected group, followed by participants in remission and, finally, by unaffected participants (from the general population and unaffected patient's relatives).

In accordance to those findings, the current study goal is to explore how different populations (healthy controls, participants with active psychotic schizophrenia, participants with schizophrenia in remission and patient's relatives) react to paranoid ideation, and examine the different aspects of their reactions (in the cognitive, behavioral and emotional dimensions). Specifically, the authors set out to determine if (a) clinical patients and undiagnosed individuals differ in terms of their reaction to paranoid thoughts and beliefs, and (b) if this difference in paranoid reaction is associated with more disturbing experiences and feelings, as well as with the use of different (i.e. more inappropriate) coping strategies in patients with schizophrenia, when compared with undiagnosed individuals.

\section{Method}

\section{Participants and procedures}


RUNNING HEAD: Differences in reactions to paranoia

A total of 187 participants took part in the current study. From these participants, 96 were drawn from the general Azorean population (not diagnosed with any mental illness) and 91 were participants with schizophrenic psychosis, either active $(n=61)$ or in remission $(n=30)$. Participants' diagnosis was confirmed with the responsible psychiatric staff and by consulting the patients' files. Only the patients diagnosed for 6 or more months entered the clinical samples in this study and all patients were currently receiving psychiatric treatment and medication. Concerning the undiagnosed subjects, 64 were healthy controls (from the general population) and 32 were direct relatives of participants taking part in the study. Study goals were explained and informed consent was obtained from all participants who agreed to complete the self-report scales. Participants suffering from schizophrenia often require assistance with self-report scales. Therefore, to avoid potential biases due to reading or interpretation difficulties, and to assure that the questionnaires were answered correctly, a senior psychologist was present to clarify any doubts or questions, and to help with proper filling of the scales.

\section{Measures}

General Paranoia Scale - GPS $(23,31)$ is a self-report questionnaire designed to assess paranoid ideation in the general population. Items refer to the belief that other people may influence our behavior and/or that they may hold something against us in several ways. Items also refer to beliefs that may induce suspicion and the impression of being judged poorly by others. The 20 items are coded in a 5-point Likert scale and total scores can range from 20 to 100, where higher scores suggest more paranoid ideations. The GPS revealed good psychometric properties, with internal consistency ranging from .78 to .89 in normative samples (23). In the current study, Cronbach's alpha were of .92.

Paranoia Checklist- $P C(11,31)$ is a measure assessing paranoid ideation in its more severe aspects. PC was designed to assess three dimensions in clinical populations: frequency of 
RUNNING HEAD: Differences in reactions to paranoia

paranoid thoughts, the degree of conviction that they are real, and the distress caused by these thoughts. Internal consistency was high in all dimensions in the original studies (11) and for the sample in the current study ( $\alpha>.90$ for the three dimensions).

Personal Experience of Paranoia Scale - PEPS $(28,31)$ is a 15-item questionnaire developed to examine the incidence and phenomenology of paranoia in the general population in three dimensions: cognitive, behavioral and affective aspects of paranoia. The questionnaire is composed of open and closed questions, and can collect richer information relating to personal paranoid experiences. Firstly, the PEPS defines paranoia as the perception of harmful intentions from others towards the self. The respondents must then refer if they have had this experience and, if so, fill the remaining questions describing reactions that are significant to the definition of clinical paranoia. Respondents that answer "no" to the first question are classified as "not paranoid". Respondents that answer "yes" to the first item are only classified as paranoid if the remaining scores show a clear perception of harmful intents from others. If this perception is not entirely clear, respondents are classified as "ambiguous". Items refer to cognitive components of paranoia (such as beliefs about their causes, impact of paranoid thoughts in personal wellbeing, worry, frequency of paranoid thoughts, and maintenance of beliefs); affective components (feelings identified during paranoid experiences), and behavioral components. The behavioral component includes actions imagined and taken as a response to paranoid thoughts: confrontation, avoidance, rationalization (look for alternative and realistic explanations), catharsis (e.g. crying or screaming), and doing nothing (28). A study by Chadwick and cols. (43) have also evaluated the belief that one deserved to be mistreated by others $(1-$ totally undeserved to $5=$ totally deserved it).

Psychotic Symptom Rating Scale-beliefs - PSYRATS-beliefs (32, 33) was used as a multidimensional assessment of paranoid ideation and to determine the nature of individual 
RUNNING HEAD: Differences in reactions to paranoia

dimensions of psychotic symptoms. It has 2 subscales, one assessing delusional activity and another assessing auditory hallucination. The delusions subscale has 6 items rated on a 5point Likert scale, referring to different dimensions of delusional activity: degree of worry, duration of worry, degree of conviction, quantity and intensity of distress and invalidation caused by delusions. This scale has presented good reliability and validity, as well as sensitivity to change (44). In the current study, internal consistency was good $(\alpha=.88)$.

\section{Statistical Analyses}

Pearson product-moment correlation coefficients were calculated to examine relationships between the different measures of paranoia. To reduce the probability of type I errors, a multivariate analysis of variance was carried out to confirm the group effect prior to calculating multiple independent ANOVAS. Analyses of variance (with Welch robust test) and post-hoc tests to determine observed differences between groups on the aforementioned measures. Chi-Square tests were used to assess statistical differences in the nominal scales of paranoid ideation, using Fischer exact test, when applicable.

\section{Results}

\section{Sample characteristics}

The four groups differed significantly regarding schooling $\left(\mathrm{F}_{(3,164)}=8.230 ; p=.000\right)$, marital status $\left(\chi^{2}=66.975 ; p=.000\right)$, and socioeconomic status $\left(\chi^{2}=43.789 ; p=.000\right)$. Concerning the age differences $\left(\mathrm{F}_{(3,181)}=5.432 ; p=.001\right)$, post-hoc tests revealed that the difference was between the relatives of participants with schizophrenia and the remaining groups, which is due to the fact that most of the relatives of the participants with schizophrenia were their caretakers (parents or older siblings). Non-clinical and clinical groups do not differ in gender distribution $\left(\chi^{2}=.426 ; p=.560\right)$, assuring further sample comparability.

*** Insert table 1 about here*** 
RUNNING HEAD: Differences in reactions to paranoia

The results of the GPS, the PC and the PSYRATS-beliefs questionnaires were significantly correlated, with Pearson correlations ranging from $r=.45$ to $r=.80, p<.001$. These results indicate the convergence between these three scales evaluating a similar construct. Scores for all measures used are presented by group in table 2.

**Insert table 2 about here**

\section{Invalidation caused by paranoid beliefs}

Significant correlations ranging between .51 and .68 were found between ratings on the \#6 item of the PSYRATS-beliefs scale (referring to the degree invalidation caused by paranoia ideation) and results of the other measures of paranoia $(p<.005)$. These findings suggest that the higher the scores on the paranoia measures, the greater the disability resulting from paranoid ideations, and the same associations were found for items referring to the duration, amount and intensity of distress assessed by the PSYRATS-beliefs scale ${ }^{1}$. Multivariate analysis of variance revealed a significant effect of the group for the multiple items in PSYRATS-beliefs: $\mathrm{F}_{(4 ; 183)}=12.674, p=.000$. Analysis of variances with Post-hoc tests were then used to examine in which groups these differences would lie on.

Item \#6 focuses on the disability or invalidation arising from paranoid ideations, incapacitating the person to work, to care for him/herself and to perform daily tasks or harming interpersonal relationships. The ANOVA performed on this item revealed a main effect due to groups, $\mathrm{F}_{(3 ; 183)}=88.616, p=.000$. Post-hoc tests showed that paranoia induced invalidation were significantly higher among active schizophrenic participants than in other groups $(p=.000)$. Also, considering the values reported in table 3 , results obtained by the group in remission were not statistically different from healthy controls $(p=.170)$, but were significantly higher than the relatives group $(p=.034)$

\footnotetext{
${ }^{1}$ The PSYRATS-beliefs scale includes a complete description of all levels of invalidation (O- 4). The definitions used were taken from that description.
} 
RUNNING HEAD: Differences in reactions to paranoia

**Insert table 3 about here**

Main effects of group on duration of preoccupation with delusions: $F_{(3 ; 183)}=18.060$, $p=.000$ (for item \#2); amount of distress, $\mathrm{F}_{(3 ; 183)}=21.774, p=.000$ (for item \#4) and distress intensity, $\mathrm{F}_{(3 ; 183)}=33.409, p=.000$ (for item \#5) were all significant. In all three instances, active psychotic participants spent more time dwelling on paranoid thoughts, had more of these thoughts and experienced them with greater intensity $(p<.005)$ than the healthy controls or relatives. Participants in remission did not differ from unaffected groups, except for the significantly higher preoccupation with delusions $(p=.006)$.

\section{Cognitive Profile and reactions to paranoid ideation}

As reported in a previous study (30), the results obtained from GPS and PC showed that participants suffering from schizophrenia became more convinced and more distressed about their paranoid ideations than people without schizophrenia. Also, scores on the PC revealed the presence of paranoid thoughts in all groups. To understand the extent to which individuals in each of the 4 groups experience paranoid thoughts and how they react to them, statistical analyses proceeded based on PEPS results concerning the cognitive, affective and behavioral reactions to paranoid thoughts.

Ratings on question \#1 of the PEPS (and further items, when applicable) were analyzed according to in order to establish whether subjects' were classified as being paranoid, non-paranoid or ambiguous. Multivariate analysis of variance revealed a significant effect of the item \#1 for the multiple paranoia measures (GPS, PC and PSYRATSbeliefs): $\mathrm{F}_{(2 ; 184)}=10.195, p=.000$. Next, to test differences in results between the PEPS and the other scales assessing paranoid ideation, an analysis of variance was performed to examine whether there were any significant differences between the PEPS classifications, and scores on the Paranoia Scales, taken separately as dependent variables. ANOVA results 
RUNNING HEAD: Differences in reactions to paranoia

showed significant results for all variables entered $\left[\mathrm{F}_{(2,184)}=43.042, p=.000\right.$ with GPS; $\mathrm{F}$ $(2,184)=33.346, p=.000$ with PC Frequency; $F_{(2,184)}=22.125, p=.000$ with PC Conviction.; $\mathrm{F}$ $(2,184)=21.739, p=.000$ with PC Distress.; $F_{(2,177)}=36.113, p=.000$ with PSYRATS-beliefs. Post-hoc tests confirm that the paranoid group scored significantly higher on all measures of paranoia than both the ambiguous and the non-paranoid group $(p<.001)$, with the latter group presenting the lowest scores on the different scales of paranoia used in this study.

Results also showed that individuals with paranoia, classified by PEPS, were present in all four groups, including the unaffected groups (healthy controls $n=14 ; 21.9 \%$; Active psychotic participants $n=43 ; 70.5 \%$; Participants in remission $n=15,50 \%$; Patient's relatives $\mathrm{n}=2 ; 6.2 \%$ ). Non-paranoid participants were removed from further analysis. Because only 8 individuals were classified as ambiguous, these subjects were also removed, as a conservative step to avoid including reports of episodes that may be related to social anxiety (28).

To evaluate the cognitive profile of individuals with paranoia $(n=72)$, the situations that were more endorsed by participants on PEPS were classified according to the categories suggested in PEPS' literature: rejection by one person, rejection by more than one person, being thwarted by one person, and being thwarted by more than one person (with the assumption of an intentional blockage in the prosecution of one's goals).

**Insert table 4 about here**

The reasons given by subjects for their paranoid ideations did not statistically differ between groups $\left(\chi^{2}(9,63)=10.883 ; p=.204\right)$ However, when participants with active schizophrenia are compared with undiagnosed individuals, the former group appears to be more sensitive to rejection by one and by more than one person (both categories correspond to $52 \%$ of the paranoia-inducing situations) than the general population (for whom these same categories correspond to $25 \%$ of the induced paranoia). Conversely, healthy controls 
RUNNING HEAD: Differences in reactions to paranoia

became more paranoid when thwarted by one or more than one person. These two latter categories constitutes $75 \%$ paranoia-inducing situations in this group, as opposed to only $48 \%$ in the group of active psychosis participants (see table 4).

Contrary to the aforementioned findings, significant differences between groups $\left(\chi_{(15,57)}^{2}=37.018 ; p=.000\right)$ were found for feelings and thoughts as cognitive triggers of paranoid ideations. However, many participants from the clinical groups were unable to determine which types of situations trigger their paranoid ideations $(n=17 ; 39,5 \%)$. Those who did usually indicate loss of control (caused by the disease itself) as the most important reason $(\mathrm{n}=13 ; 30.2 \%)$. Participants in the unaffected groups refer mainly situations of (perceived) injustice $(\mathrm{n}=10 ; 83.3 \%)$.

An analysis of variance was used to test for significant differences in the conviction of individuals regarding intention to harm from others. The factor involved in this analysis was question \#3 of the PEPS ("how much did you believe it?") and the dependent variable was the responses of subjects across groups. The degree of conviction on the intent to harm from others differed significantly across groups, $\mathrm{F}_{(3,68)}=3.160, p=.030$. Post-hoc tests reveal that the degree of conviction of healthy controls is significantly lower than the conviction of participants in remission $(p=.033)$. Except for this difference, these convictions were found to be stable across groups.

Similarly, no statistically significant differences $(p>.005)$ were found between groups regarding (1) levels of apprehension about paranoid thoughts $\left[\mathrm{F}_{(3 ; 68)}=1.618, p=.193\right]$; (2) the impact that these thoughts have on the participants' everyday lives $\left[\mathrm{F}_{(3 ; 68)}=.904, p=.444\right]$; and (3) the degree to which participants believe that they deserve to be ill-treated by others $\left[\mathrm{F}_{(3 ; 68)}=.548, p=.651\right] ;$ as rated in items $\# 9, \# 11$ and $\# 13$ of the PEPS, respectively.

Concerning participant's behavior (reactions) when confronted with paranoid beliefs, there were statistically significant differences between observed and expected frequencies in 
RUNNING HEAD: Differences in reactions to paranoia

the responses of subjects across groups $\left[\chi_{(3,69)}^{2}=14.380 ; p=.001\right]$. Results show that both healthy controls and patient's relatives reacted to paranoid thoughts (unaffected controls $n=$ $8 ; 66.7 \%$; relatives $n=2,100 \%$ ), while most participants with active psychosis failed to do so $(n=35,81.4 \%)$. Differences in the maintenance of paranoid beliefs were found, although very close to significance threshold, indicating that the relatives group tends to reevaluate their paranoid thoughts while the remaining groups tend to hold to their paranoid interpretations: $\left[\chi_{(3,69)}^{2}=7.275 ; p=.046\right]$. No differences were found between subjects who changed their minds about their paranoid beliefs, although a tendency to resource to rationalization was present $\left(\chi_{(3,69)}^{2}=8.828 ; p=.082\right)$ (see table 5).

**Insert table 5 about here**

As far as variations in the types of reactions to paranoia inducing situations are concerned, no statistically significant differences in group results were found in this regard, $\chi^{2}$ $(15,57)=18.049 ; p=.237$. However, some trends are worth considering: unaffected groups' ratings involved exclusively confrontational responses (healthy controls $n=5,63.5 \%$; relatives $\mathrm{n}=2,100 \%$ ) while active psychotic participants' endorsements ranged between avoidance $(n=2,34.3 \%)$, catharsis $(n=2,34.3 \%)$ and confrontation $(n=4,28.6 \%)$. The latter group also reacted less to paranoia inducing situations. Just about $28 \%$ of the active psychotic participants reacted at all, as compared, for example, with their unaffected relatives $(100 \%)$, or with subjects from the general population (62.5\%) that were classified as paranoid.

\section{Discussion}

The purpose of the current study was to examine the profile of the cognitive, behavioral and affective components of participants' experiences and reactions to paranoid beliefs. Differences in terms of reactions to paranoid beliefs were expected, even though 
RUNNING HEAD: Differences in reactions to paranoia

paranoia is a shared thought process across all individuals. Results showed that paranoia was present across the four groups, as measured by the PEPS and in consonance current findings on the continuity of paranoid ideation $(1-11,30)$. That is, all individuals presented, to some extent, convictions about others' intentions to harm them. This finding of the existence of paranoia in the general population $(6.2 \%$ for relatives and $21.9 \%$ for controls $)$ reinforces the idea of the continuity in paranoid ideations in clinical to subclinical levels in previous research $(1-6,11,12,23,24)$.

Further results showed that, in the schizophrenic group, some individuals were classified as free of paranoid symptoms $(27.9 \%$ of active schizophrenics and $50 \%$ of participants in remission). The existence of subjects without paranoia in both clinical groups may be explained by the use of antipsychotic medication and psychiatric intervention, which decreases paranoid delusions to more residual levels. In the active group, the number of subjects without paranoia is smaller than in the remission group (as the state of decompensation caused by the disease does not necessarily involve the presence of paranoid delusion). Interestingly, results showed that participants with active schizophrenic psychosis were not as convinced about their own perceptions regarding planned intentions to harm from others as the participants in remission and, surprisingly, they did not differ from healthy controls and unaffected relatives. This may be due to the fact that adopting a defensive stance to a perceived threat is a normal and adaptive response and, once a paranoid belief is triggered, the degree of conviction in the paranoid ideation is the same regardless of the participant's condition. Thus, the main difference between affected and non-affected individuals may rely predominantly on the threshold and frequency with which these beliefs are triggered.

One of the most important aspects in the assessment of the continuity of paranoid beliefs, and a distinctive feature between the clinical and the nonclinical population, is the 
RUNNING HEAD: Differences in reactions to paranoia

degree of invalidation caused by those beliefs. Our results led to the conclusion that, for the great majority of the individuals presenting paranoia, paranoid thoughts can cause severe invalidation and the need for hospitalization. One possible explanation for this severe invalidation is the time spent thinking about their paranoid ideations (accompanied by distress and concern about these beliefs). Results from this study show that active psychotic participants actually spent more time thinking about their paranoid ideations than unaffected individuals (reaching up to one hour daily). The paranoid thoughts they present are not only more frequent than in healthy people, but they also consume more of their daily cognitive activity.

Research in this area $(15,16,19,34-42)$ points out to emotional disorders associated with clinical paranoid ideations. Consistent with these findings, our results showed that active schizophrenic participants (1) become highly distressed and concerned about their paranoid beliefs, and (2) experience distress almost every time these ideations appear. Considering that the group of healthy controls may be representative of individuals without schizophrenic pathology, it is possible to state that, for most people and most of the time, paranoid thoughts are transitory experiences that cause little distress and no invalidation. However, schizophrenic patients can exacerbate reality, transforming these thoughts into more frequent episodes of anguish and anxiety, leading to more daily invalidation.

The unaffected relatives showed lower (although not statistically significant) scores on the emotional dimension when compared with healthy controls in most of the PSYRATSbeliefs scale items. This tendency can be due to their close contact with schizophrenic participants, inasmuch as the suffering of the affected individuals provides them with the opportunity to learn how to cope with paranoid thoughts. Nevertheless, relatives' ratings on this matter indicated a tendency dwell longer on these thoughts than healthy controls. The 
RUNNING HEAD: Differences in reactions to paranoia

latter group has shown to be less distressed by such beliefs and their invalidating tendencies are also less apparent.

The fact that invalidation caused by paranoid ideations does not differ significantly between psychotic participants in remission and healthy controls supports the hypothesis about the effectiveness of antipsychotic medication and treatment, as previously argued. Participants in remission would probably return to levels of invalidation similar those shown by subjects in the group of active psychotic participants, should they decompensate again.

Results concerning the cognitive profile of participants' experiences (to which only paranoid individuals have been assessed through the PEPS), showed that active schizophrenic participants appeared to be more sensitive to rejection by one person or more than one person when compared to healthy subjects who, in turn, react more to (i.e. become more paranoid in) situations where they feel that they are being thwarted by others. These differences, although not statistically significant, can indicate that (for active schizophrenic participants) the perception of intention to harm from others arises in any context of social rejection, and not only in situations where the person feels thwarted by others. This can also explain the increased frequencies of paranoid ideations which active participants experience in daily situations.

This latter conclusion was reinforced by the significant differences found between groups regarding the circumstances that induce paranoid feelings and thoughts. Injustice was the major reason for paranoid thoughts by the healthy controls. These results emphasize the defensive function of paranoid ideation as proposed by Gilbert $(43,44)$. To this author, the self-protective nature of paranoia is encompassed in the social defenses to threats adopted by individuals of the same species being, thus, related to different types of social roles. Furthermore, considering previous findings on the frequency and resource-consuming cognitive activity related to paranoid ideation, as a part of a defense to threat system, 
RUNNING HEAD: Differences in reactions to paranoia

paranoia can activate a "better safe than sorry" mindset to social interactions. This means that, in socially threatening environments, it is better to presume that things can be threatening than to fall short of recognizing the real threat. The more frequent the paranoid thoughts, the greater the threat system can affect information processing capabilities in the individual.

However, the reader should bear in mind that most participants (both active and in remission) were unable to ascertain situations that induce paranoid ideations, and loss of control (caused by the disease itself) was the most important reason for those who could identify them. When active psychotic participants refer the rejection by others as the most important activator of their paranoid ideations, but cannot identify the situations that trigger such thoughts, it is possible that they find themselves in situations where the perceptions of threats (associated with paranoid ideations) are more easily triggered. This may occur for different reasons: As Freeman et al. (13) suggested, schizophrenic participants can have a paranoid scheme, or bias, for processing information, since they are sensitive to ambiguous social stimuli, which will influence the processing of the stimulus as threatening "by default" $(3,13,17,45)$, activating a "better safe than sorry" approach to interpersonal relationships. On the other hand, the perception of loss of control referred by some participants reinforces Freeman's idea that persecutory delusions are more likely to be presented by individuals who see themselves as vulnerable - or as "an easy target" (46).

This greater probability associated with the activation of paranoid beliefs seems to be one of the most important differentiating factors between clinical and nonclinical paranoid ideations. It should be noted that, in the current study, clinical and nonclinical subjects showed no statistically significant differences insofar as the presence of paranoid thoughts are concerned. In addition, the perseverance of paranoid thoughts presented by the participants revealed not only that paranoid ideation are resistant to change, but they occur over time 
RUNNING HEAD: Differences in reactions to paranoia

regardless of the existence of the schizophrenic pathology. The great majority of participants classified as paranoid maintained their initial thoughts, showed their concern about them, and referred a considerable impact of these beliefs on their everyday lives. Changes were reported only by a minority of subjects (in both the unaffected and participants in remission groups) and were justified through rationalization. However, although more conservative and robust tests were used, these results should be interpreted and generalized carefully, considering the number of subjects in each sample.

The most significant results obtained in this study involve the differences found between the group of active psychotic participants and the healthy controls on the cognitive behavior profile. As stated by Freeman, et al. (11), high levels of paranoia are more associated with emotional and avoidant coping strategies than with rational strategies. Findings from the current study are consistent with current research inasmuch as the unaffected groups (healthy controls and relatives) report reacting to the occurrence of paranoid beliefs, while active participants generally state that they do not react to such thoughts (with participants in remission report experiencing both situations). As far as the nature of their reactions is concerned, there is a qualitative difference between the responses of those who report confronting the situation (unaffected groups) and those who report mainly avoiding and catharsis as a means of coping with it (clinical groups). The authors consider that, by not reacting, most of the paranoia active participants fail to disconfirm their paranoid beliefs. Consequently, by dwelling on these thoughts, increased amounts of time are also spent thinking about their condition.

This research is not free from limitations. Generalization of findings should be done carefully, taking into consideration to the small sample sizes and cross-sectional design of the current study. Future studies should explore the influence of familial, emotional and social variables in the development and manifestation of paranoid ideation in different populations 
RUNNING HEAD: Differences in reactions to paranoia

(e.g. Prediction studies). Possible changes in these reactions to paranoia may present over time and according to patient's current condition (cognitive, emotional and behavioral reactions when patients are in active or compensated state, for example) should also be further addressed in longitudinal studies.

Results from this study support the idea of a clinical approach to the treatment of paranoia with a cognitive focus, and aimed at the development of capabilities of insight and reassessment of individual experiences that can boost paranoid interpretations of reality. This approach is deemed as very promising, as it can be a real "lifeline" for participants who, in the past, have been deprived of the therapeutic resources for dealing with a chronic and very disabling disease like schizophrenia.

\section{References}

1. Verdoux H \& van Os J. Psychotic symptoms in non-clinical populations and the continuum of psychosis. Schizophr Res, 2002, Mar 1;54(1-2):59-65. DOI: 10.1016/S0920-9964(01)00352-8.

2. Combs D, Michael C \& Penn D. Paranoia and emotion perception across the $\begin{array}{lllll}\text { continuum. } & \mathrm{Br} & \mathrm{J} & \mathrm{Clin} & \text { Psychol. } 2006 \quad \text { Mar;45(1):19-31. DOI: }\end{array}$ $10.1348 / 014466505 X 29099$.

3. Campbell M \& Morrison A (2007). The Subjective Experience of Paranoia: Comparing the Experiences of Patients with Psychosis and Individuals with No Psychiatric History. Clin. Psychol. Psychother. 2007 Jan 29;14(1), 63-77 DOI: 10.1002/cpp.510.

4. van Os J, Linscott RJ, Myin-Germeys I, Delespaul P \& Krabbendam L. A systematic review and meta-analysis of the psychosis continuum: evidence for a psychosis proneness-persistence-impairment model of psychotic disorder. Psychol Med. 2009 Feb;39(2):179-95. doi: 10.1017/S0033291708003814. 
RUNNING HEAD: Differences in reactions to paranoia

5. Melo, S \& Bentall, R. Coping in subclinical paranoia: A two nations study. Psychol Psychother. 2010 Mar 12; 83(4), 407-420; DOI: 10.1348/147608310X487542.

6. Michel C, Schaffner N, Shimmelmann B. \& Shultze-Lutter F. What percentage of people in the general population satisfies the current clinical at-risk criteria of psychosis? Schizophr $\quad$ Res. $2011 \quad$ Jan;125(1):99-100. doi: 10.1016/j.schres.2010.09.018

7. Garety P \& Hemsley D. Delusions: Investigations into the psychology of delusional reasoning. Oxford: Oxford University Press; 1994.

8. Fenigstein A. Paranoid thought and schematic processing. J Soc Clin Psychol, 1997; 16: 77-94. DOI: $10.1521 /$ jscp.1997.16.1.77.

9. Garety, P. \& Freeman, D. (1999). Cognitive approaches to delusions: a critical review of theories and evidence. Br J Clin Psychol. 1999 Jun;38 (Pt2):113-54. DOI: 10.1348/014466599162700.

10. Combs D, Penn D. \& Fenigstein A. Ethnic Differences in Subclinical Paranoia: An Expansion of Norms of the Paranoia Scale. Cultur Divers Ethnic Minor Psychol. 2002 Aug;8(3):248-56. DOI: 10.1037/1099-9809.8.3.248.

11. Freeman D, Garety PA, Bebbington, PE, Smith B, Rollinson R, Fowler D, Kuipers E., Ray K. \& Dunn G. Psychological investigation of the structure of paranoia in a nonclinical population. $\mathrm{Br} \quad \mathrm{J}$ Psychiatry, 2005;186:427-435. DOI: : 10.1192/bjp.186.5.427.

12. Martin, J. \& Penn D. (2001). Social cognition and subclinical paranoid ideation. Br J Clin Psychol. 2001 Sep;40(3):261-5. DOI: 10.1348/014466501163670.

13. Freeman D, Garety P, Kuipers E, Fowler D \& Bebbington, E. A Cognitive model of persecutory delusions. $\mathrm{Br} \quad \mathrm{J}$ Clin Psychol. 2002 Nov;41(4):331-47. DOI: $10.1348 / 014466502760387461$. 
RUNNING HEAD: Differences in reactions to paranoia

14. Bentall R, Kinderman P \& Kaney S. The self, attributional processes and abnormal beliefs: Towards a model of persecutory delusions. Behav Res Ther. 1994 Mar;32(3):331-41.. DOI: 10.1016/0005-7967(94)90131-7.

15. Kinderman P \& Bentall R. Self-discrepancies and persecutory delusions: Evidence for a model of paranoid ideation. J Abnorm Psychol. 1996 Feb;105(1):106-13. DOI: 10.1037/0021-843X.105.1.106.

16. Bentall R, Kaney S \& Bowen-Jones K. Persecutory delusions and recall of threat related, depression related, and neutral words. Cognit Ther Res, Aug, 1995;19: 445457. DOI: $10.1007 / \mathrm{BF} 02230411$.

17. Phillips M, Senior C \& Davis A. Perception of threat in schizophrenics with persecutory delusions: An investigation using visual scan paths. Psychol Med. 2000 Jan;30(1):157-67.

18. Tait L, Birchwood M, \& Trower P. Adapting to the challenge of psychosis: personal resilience and the use of sealing-over (avoidant) coping strategies. $\mathrm{Br} \mathrm{J}$ Psychiatry. 2004 Nov;185:410-5.. DOI: 10.1192/bjp.185.5.410.

19. Baldwin M \& Dandeneau S. Interpersonal Cognition. Baldwin MW (Ed). New York: Guilford; 2005. Understanding and modifying schemas underlying insecurity; p. 3361.

20. Freeman D, Pugh K. \& Garety P. Jumping to conclusions and paranoid ideation in the general population. Schizoph Res. 2008;102(1-3):254-60. doi:10.1016/j.schres.2008.03.020.

21. Georgaca E. (2004).Talk and the Nature of Delusions: Defending Sociocultural Perspectives on Mental Illness. Philos Psychiatr Psychol. 2004: 11(1):87-94. DOI: 0.1353/ppp.2004.0038. 
RUNNING HEAD: Differences in reactions to paranoia

22. Beck AT \& Rector N. Cognitive Approaches to Schizophrenia: Theory and Therapy. $\begin{array}{llll}\text { Annu } & \text { Rev } & \text { Psychol. 2005;1:577-606. DOI: }\end{array}$ 10.1146/annurev.clinpsy.1.102803.144205.

23. Fenigstein A \& Vanable P. (1992). Paranoia and self-consciousness. J Pers Soc Psychol. 1992 Jan;62(1):129-38.DOI: 10.1037/0022-3514.62.1.129.

24. Strauss J. Hallucinations and delusions as points on continua functions. Arch Gen Psychiatry. 1969;21(5):581-586. doi:10.1001/archpsyc.1969.01740230069010

25. Strauss J. Subjective experiences of schizophrenia: Toward a new dynamic psychiatry II. Schizophr Bull. 1989;15(2):179-87. DOI: 10.1093/schbul/15.2.179.

26. Eaton, WW, Romanoski A, Anthony JC \& Nestadt G. Screening for psychosis in the general population with a self-report interview. J Nerv Ment Dis. 1991 Nov;179(11):689-93. DOI: 10.1097/00005053-199111000-00007.

27. van Os J., Hanssen M., Bijl RV \& Ravelli A. Strauss (1969) revisited: a psychosis continuum in the general population? Schizophr Res. 2000 Sep 29;45(1-2):11-20.. DOI: 10.1016/S0920-9964(00)90323-2.

28. Ellett L, Lopes B, \& Chadwick P. Paranoia in a Nonclinical Population of College $\begin{array}{lllll}\text { Students. J Nerv Ment Dis. } 2003 \text { Jul;191(7):425-30. DOI: } & \end{array}$ 10.1097/01.NMD.0000081646.33030.EF.

29. Freeman D., McManus S, Brugha T, Meltzer H, Jenkins R. \& Bebbington P. Concomitants of paranoia in the general population. Psychol Med. 2011 May;41(5):923-36. doi: 10.1017/S0033291710001546

30. Barreto Carvalho C., Pinto-Gouveia J, Peixoto E. \& da Motta C. Paranoia as a continuum in the population. Manuscript submitted for publication; 2014.

31. Lopes B. Paranoia e Ansiedade Social na População Não-Clínica [Dissertation]. Coimbra: University of Coimbra;2010. 285p. 
RUNNING HEAD: Differences in reactions to paranoia

32. Haddock C, McCarron J, Tarrier N, \& Faragher EB (1999). Scales to measure dimensions of hallucinations and delusions: the Psychotic Symptom Rating Scales (PSYRATS). Psychol Med. 1999 Jul;29(4):879-89.

33. Barreto Carvalho C, \& Pinto-Gouveia, J (2008) PSYRATS-beliefs - Portuguese adaptation of Haddock et. al, 1999; 2008.

34. Adams, HE Malatesta V, Brantley PJ \& Turkat ID. Modification of cognitive processes: A case study of schizophrenia. J Consult Clin Psychol. 1981 Jun;49(3):460-4.

35. Hatfield AB. Participants' accounts of stress and coping in schizophrenia. Hosp Community Psychiatry. 1989; 40:1141-1145

36. Hole RW, Rush AJ, \& Beck AT. A cognitive investigation of schizophrenic delusions. Psychiatry. 1979 Nov;42(4):312-9.

37. Clements K, \& Turpin G. Innovations in the psychological management of schizophrenia: Assessment, treatment and services. Chichester: Wiley; 1992. Vulnerability models and schizophrenia: The assessment and prediction of relapse; pp. 21-47.

38. Alford, BA \& Beck AT. The Integrative Power of Cognitive Therapy. New York: Guilford Press; 1997. Schizophrenia and Other Psychotic Disorders pp. 137-164.

39. Fowler, D. Early intervention in psychosis: A guide to concepts, evidence and interventions. Chichester: Wiley; 2000. Psychological formulation of early episodes of psychosis: a cognitive model; pp. 101-127.

40. Dagnan D, Trower P \& Gilbert P. Measuring vulnerability to threats to selfconstruction: The self and other Scale. Psychol Psychother. 2002 Sep;75(3):279-93. DOI: $10.1348 / 147608302320365271$. 
RUNNING HEAD: Differences in reactions to paranoia

41. Freeman D \& Garety P. Connecting neurosis and psychosis: The direct influence of emotion on delusions and hallucinations. Behav Res Ther. 2003 Aug;41(8):923-47. DOI: $10.1016 / \mathrm{S} 0005-7967(02) 00104-3$.

42. Chadwick P, Trower P, Juusti-Butlerb, TM \& Maguirea N. (2005). Phenomenological Evidence for Two Types of Paranoia. Psychopathology. 2005 Nov-Dec;38(6):327-33 DOI: $10.1159 / 000089453$.

43. Gilbert P. Evolutionary psychopathology: Why isn't the mind designed better than it is? $\quad \mathrm{Br} \quad \mathrm{J} \quad$ Med Psychol. $1998 \quad$ Dec;71(4):353-73. DOI: 10.1111/j.20448341.1998.tb00998.x.

44. Gilbert P., Boxall, M., Cheung, M. \& Irons, C. (2005). The Relation of Paranoid Ideation and Social Anxiety in a Mixed Clinical Population. Clinical Psychol Psychother. 2005 Apr:12:125-133. DOI: 10.1002/cpp.438.

45. Miller R \& Mason S. Shame and Guilt in First-Episode Schizophrenia and Schizoaffective Disorders. J Contemp Psychother. 2005;35(2):211-221. DOI: $10.1007 / \mathrm{s} 10879-005-2701-4$.

46. Freeman D, Garety P, Fowler D, Kuipers E, Dunn G, Bebbington, E \& Hadley C. The London-East Anglia randomized controlled trial of cognitive behaviour therapy for psychosis. IV: Self-esteem and persecutory delusion. Br J Clin Psychol. 1998 Nov;37 (4):415-30.

\section{Acknowledgements}

The authors would like to thank the cooperation from the Psychiatric Services from the Hospital Divino Espirito Santo, and the staff from Casa de Saúde Nossa Senhora da Conceição and Casa de Saúde São Miguel. The authors would also like to thank Marina Sousa for preparing the manuscript. 
Table 1 - Sample Characteristics $(n=187)$

\begin{tabular}{|c|c|c|c|c|c|c|}
\hline \multirow[b]{2}{*}{ Variables } & \multicolumn{2}{|c|}{ Non clinical sample } & \multicolumn{2}{|c|}{ Clinical sample } & & \\
\hline & $\begin{array}{c}\text { Healthy } \\
\text { controls }(\mathrm{n}= \\
64)\end{array}$ & $\begin{array}{l}\text { Participants' } \\
\text { relatives }(n= \\
32)\end{array}$ & $\begin{array}{c}\text { Active } \\
\text { psychotic }(\mathrm{n}= \\
61)\end{array}$ & $\begin{array}{l}\text { In remission (n } \\
\quad=30)\end{array}$ & & \\
\hline Gender & $\mathrm{N}(\%)$ & $\mathrm{N}(\%)$ & $\mathrm{N}(\%)$ & $\mathrm{N}(\%)$ & $\chi^{2}$ & $p$ \\
\hline Male & $43(67.2 \%)$ & $8(25 \%)$ & $20(32.8 \%)$ & $24(80 \%)$ & \multirow{2}{*}{58.94} & \multirow{2}{*}{.000} \\
\hline Female & $21(32.8 \%)$ & $24(75 \%)$ & $41(67.2 \%)$ & $6(20 \%)$ & & \\
\hline \multicolumn{7}{|l|}{ Marital status } \\
\hline Single & $18(30 \%)$ & $0(.0 \%)$ & $33(55.9 \%)$ & $21(70 \%)$ & \multirow{5}{*}{70.8} & \multirow{5}{*}{.000} \\
\hline Married & $35(58.3 \%)$ & $28(90.3 \%)$ & $13(22 \%)$ & $6(20 \%)$ & & \\
\hline Divorced & $3(5.0 \%)$ & $0(.0 \%)$ & $7(11.9 \%)$ & $2(6.7 \%)$ & & \\
\hline Widower & $3(5.0 \%)$ & $3(9.7 \%)$ & $4(6.8 \%)$ & $1(3.3 \%)$ & & \\
\hline Civil union & $0(.0 \%)$ & $0(.0 \%)$ & $1(1.7 \%)$ & $0(.0 \%)$ & & \\
\hline \multicolumn{7}{|l|}{ Socioeconomic status } \\
\hline Low & $22(37.3 \%)$ & $15(62.5 \%)$ & $46(80.7 \%)$ & $27(90 \%)$ & \multirow{4}{*}{46.60} & \multirow{4}{*}{.000} \\
\hline Medium & $20(33.9 \%)$ & $4(16.7 \%)$ & $11(19.3 \%)$ & $3(10 \%)$ & & \\
\hline High & $12(20.3 \%)$ & $5(20.8 \%)$ & $0(.0 \%)$ & $0(.0 \%)$ & & \\
\hline \multirow[t]{2}{*}{ Student } & $5(8.5 \%)$ & $0(.0 \%)$ & $0(.0 \%)$ & $0(.0 \%)$ & & \\
\hline & $\mathrm{M}(\mathrm{SD})$ & $\mathrm{M}(\mathrm{SD})$ & $\mathrm{M}(\mathrm{SD})$ & $\mathrm{M}(\mathrm{SD})$ & $F$ & $p$ \\
\hline Age (years) & $45.2(17.3)$ & $55.6(13.0)$ & $43.8(12.6)$ & $43.5(12.6)$ & $4.53(4 ; 180)$ & .002 \\
\hline Years of schooling (years) & $9.8(4.8)$ & $7.7(4.3)$ & $6.4(3.5)$ & $6.3(3.4)$ & $6.16(4 ; 163)$ & .000 \\
\hline
\end{tabular}


Table 2. Scores for all groups on measures of paranoia (PC, GPS, PSYRATS-beliefs and PEPS)

\begin{tabular}{|c|c|c|c|c|c|c|c|c|c|c|c|c|c|c|c|c|}
\hline & \multicolumn{4}{|c|}{ Healthy controls $(\mathrm{n}=64)$} & \multicolumn{4}{|c|}{ Active psychotic $(\mathrm{n}=61)$} & \multicolumn{4}{|c|}{ In remission $(\mathrm{n}=30)$} & \multicolumn{4}{|c|}{ Patient's relatives $(\mathrm{n}=32)$} \\
\hline & $M$ & $S D$ & Min. & Max. & $M$ & $S D$ & Min. & Max. & $M$ & $S D$ & Min. & Max. & $M$ & $S D$ & Min. & $\operatorname{Max}$. \\
\hline PC Frequency & 25.16 & 11.41 & 18 & 75 & 52.05 & 19.98 & 18 & 90 & 36.43 & 19.32 & 18 & 85 & 23.59 & 10.36 & 18 & 64 \\
\hline PC Conviction & 37.13 & 21.66 & 18 & 90 & 57.70 & 17.51 & 18 & 86 & 42.53 & 19.42 & 18 & 86 & 29.44 & 14.43 & 18 & 90 \\
\hline PC Distress & 16.95 & 17.14 & 0 & 58 & 36.23 & 20.14 & 0 & 72 & 28.87 & 20.21 & 0 & 67 & 11.41 & 12.23 & 0 & 45 \\
\hline GPS & 41.55 & 12.36 & 20 & 77 & 57.85 & 15.74 & 20 & 86 & 48.40 & 16.71 & 23 & 86 & 37.25 & 10.18 & 22 & 63 \\
\hline PSYRATS & 5.75 & 4.716 & 0 & 21 & 15.36 & 5.347 & 0 & 24 & 9.93 & 5.265 & 3 & 24 & 6.03 & 3.746 & 0 & 13 \\
\hline
\end{tabular}


Table 3- ANOVA of PSYRATS-beliefs Items \#2, \#4, \#5 and \#6 analyzed across groups

\begin{tabular}{|c|c|c|c|c|c|c|c|c|c|c|}
\hline & \multicolumn{2}{|c|}{ Healthy Controls $(\mathrm{n}=64)$} & \multicolumn{2}{|c|}{ Active Psychotic $(\mathrm{n}=61)$} & \multicolumn{2}{|c|}{ In remission $(\mathrm{n}=30)$} & \multicolumn{2}{|c|}{ Patient's relatives $(n=32)$} & \multirow[b]{2}{*}{$F$} & \multirow[b]{2}{*}{$p$} \\
\hline & M & SD & M & SD & M & SD & M & SD & & \\
\hline Psyrats \#2 & 1.233 & .126 & 2.509 & .129 & 1.967 & .184 & 1.355 & .178 & 18.060 & .000 \\
\hline Psyrats \#4 & .667 & .153 & 2.271 & .156 & 1.000 & .223 & .419 & .216 & 21.774 & .000 \\
\hline Psyrats \#5 & .667 & .139 & 2.339 & .142 & .900 & .203 & .323 & .196 & 33.409 & .000 \\
\hline Psyrats \#6 & .250 & .118 & 2.695 & .121 & .800 & .173 & .064 & .168 & 88.616 & .000 \\
\hline
\end{tabular}


Table 4. Chi-square tests for paranoia-inducing situations (PEPS item \#2b) across groups

\begin{tabular}{|c|c|c|c|c|c|c|c|c|c|c|}
\hline & \multicolumn{2}{|c|}{ Healthy Controls $(\mathrm{n}=64)$} & \multicolumn{2}{|c|}{ Active Psychotic $(\mathrm{n}=61)$} & \multicolumn{2}{|c|}{ In remission $(\mathrm{n}=30)$} & \multicolumn{2}{|c|}{ Patient's relatives $(\mathrm{n}=32)$} & \multirow[t]{2}{*}{$\chi^{2}$} & \multirow[t]{2}{*}{$p$} \\
\hline & $\mathrm{N}$ & $\%$ & $\mathrm{~N}$ & $\%$ & $\mathrm{~N}$ & $\%$ & $\mathrm{~N}$ & $\%$ & & \\
\hline Rejection by one person & 2 & 16.7 & 3 & 7 & 1 & 6.7 & 0 & 0 & & \\
\hline Rejection by more than one person & 1 & 8.3 & 19 & 44.2 & 6 & 40 & 1 & 50 & 10.883 & .204 \\
\hline Thwarted by one person & 1 & 8,3 & 7 & 16.3 & 2 & 13.3 & 1 & 50 & & \\
\hline Thwarted by more than one person & 8 & 66.7 & 14 & 32.6 & 6 & 40 & 0 & 0 & & \\
\hline
\end{tabular}


Table 5. Chi-square tests for paranoia-inducing situations (PEPS item \#4) across groups

\begin{tabular}{|c|c|c|c|c|c|c|c|c|}
\hline & \multicolumn{2}{|c|}{ Healthy Controls $(\mathrm{n}=64)$} & \multicolumn{2}{|c|}{ Active Psychotic $(\mathrm{n}=61)$} & \multicolumn{2}{|c|}{ In remission $(\mathrm{n}=30)$} & \multicolumn{2}{|c|}{ Patient's relatives $(n=32)$} \\
\hline & $\mathrm{N}$ & $\%$ & $\mathrm{~N}$ & $\%$ & $\mathrm{~N}$ & $\%$ & $\mathrm{~N}$ & $\%$ \\
\hline No & 10 & 83.3 & 37 & 86 & 11 & 73.3 & 0 & 0 \\
\hline Yes & 2 & 16.7 & 6 & 14 & 4 & 26.7 & 2 & 100 \\
\hline \multicolumn{9}{|l|}{ Why did it change? } \\
\hline Doesn’t know & 0 & 0 & 2 & 33.3 & 0 & 0 & 2 & 100 \\
\hline Changed the belief & 0 & 0 & 2 & 33.3 & 0 & 0 & 0 & 0 \\
\hline Rationalization & 2 & 100 & 2 & 33.3 & 4 & 100 & 0 & 0 \\
\hline
\end{tabular}

